\title{
Resolvins: Immunoregulatory role in periodontal disease
}

\author{
Rajiv Saini* \\ Department of Periodontology \& Oral Implantology, Pravara Institute of Medical Sciences-Loni, Maharashtra, India
}

\section{Editorial}

Periodontal diseases are recognized as infectious processes that require bacterial presence and a host response and are further affected and modified by other local, environmental, and genetic factors. Association of periodontal infection with organ systems like cardiovascular system, endocrine system, reproductive system, and respiratory system makes periodontal infection a complex multiphase disease. Inflamed periodontal tissues produce significant amounts of pro-inflammatory cytokines, particularly, inflammatory cytokines, such as IL-l $\alpha, 1 \mathrm{~L}-1 \beta$, IL-6, and IL-8, are present in the diseased periodontal tissues, and their unrestricted production seems to play a role in chronic leukocyte recruitment and tissue destruction. Acute periodontal disease primarily involves a local innate immune response to the microflora of the oral biofilm. Gingival epithelial cells recognize bacterial cell components via toll-like receptors and respond by producing IL-1 and TNF- $\alpha$. Bacteria and bacterial products also penetrate into the underlying tissues. There they interact with fibroblasts and dendrite cells. These cells also produce pro-inflammatory cytokines [1].

They are compounds that are made by the human body from the omega-3 fatty acids, eicosapentaenoic acid (EPA) and docosahexaenoic acid (DHA). Compounds derived from EPA are designated as Resolvins of the E series (RvE1), and those biosynthesized from DHA are denoted as Resolvins of the D series (RvD). Resolvins stimulate the resolution of inflammation through multiple mechanisms, including preventing neutrophil penetration, phagocytosing apoptotic neutrophils to clear the lesion, and enhancing clearance of inflammation within the lesion to promote tissue regeneration [2-4].

The local response to acute inflammation is an active rather than a passive process that triggers specific biochemical and cellular programs of resolution. The first "pro-resolution" pathway involves novel lipid mediators that possess endogenous anti-inflammatory and pro-resolution properties that are termed as Rv. In addition, novel lipid mediators produced from DHA, an abundant omega- 3 fatty acid in neural tissues, are now coined as docosatrienes, and $17 \mathrm{~S}$ series $\mathrm{Rv}$ were found to be both anti-inflammatory and tissue protective [5]. In the Porphyromonas gingivalis-induced rabbit model of periodontitis RvE1 prepared by total organic synthesis was topically applied to $P$. gingivalis ligatured teeth $(4 \mu \mathrm{g} / \mathrm{tooth}) 3$ times a week in the rabbit model over a 6-week study period. Control groups received the same frequency of topical application of vehicle (ethanol) together with $P$. gingivalis application to ligatured teeth or placement of ligature alone. [2] In a $P$. gingivalis-induced rabbit model of experimental periodontitis, RvE1 was potent when topically applied, demonstrating remarkable efficacy in the prevention and treatment of periodontal disease. As such, targeted agents, such as RvE1, that stimulate the resolution of inflammation may offer some therapeutic advantage in the treatment of periodontitis compared to more traditional pharmacologic interventions [6].
Resolvins stimulate the resolution of inflammation through multiple mechanisms, including preventing neutrophil penetration, phagocytosing apoptotic neutrophils to clear the lesion, and enhancing clearance of inflammation within the lesion to promote tissue regeneration.

A previous study indicated that topical application of RvE1 to rabbit periodontal tissue conferred dramatic protection against tissue and bone loss associated with periodontitis. In that study, it was also demonstrated that PMNs from localized aggressive periodontitis were refractory to resolving molecules of the lipoxin series. However, PMNs responded to RvE1, which stopped superoxide anion generation by binding at a site that is functionally distinct from the aspirin-triggered lipoxin receptor. These studies revealed the potential of using resolvins for prevention and treatment of periodontal disease. Furthermore, they provide a new role for resolvin signaling in the pathogenesis of periodontal disease [7].

A large body of recent work suggests that the resolvins, associated with inflammation, are the molecules responsible for the resolution of inflammation. These molecules have been demonstrated to be important in a variety of disease processes, and their therapeutic potential has been identified in a variety of model systems. It has been demonstrated that resolution of inflammation in periodontitis through resolving mediated pathways offers potential for the prevention and perhaps treatment of periodontal lesions. Future studies need to focus on the applicability of resolvin therapies in humans for the prevention and treatment of the periodontal diseases. Multiple multi-centric longitudinal interventional studies in different population groups will be required to strongly prove the application of resolvins/lipoxins in resolution of inflammation in patients of periodontitis.

\section{References}

1. Saini R, Saini S, Saini SR (2011) Periodontitis: A risk for delivery of premature labor and low birth weight infants. J Nat Sc Biol Med 2: 50-52. [Crossref]

2. Hasturk H, Kantarci A, Goguet-Surmenian E, Blackwood A, Andry C, et al. (2007) Resolvin E1 regulates inflammation at the cellular and tissue level and restores tissue homeostasis in vivo. J Immunol 179:7021-7029. [Crossref]

3. Bannenberg GL, Chiang N, Ariel A, Arita M, Tjonahen E, et al. (2005) Molecular circuits of resolution: Formation and actions of resolvins and protectins. $J$ Immunol 174: 4345-4355. [Crossref]

4. Schwab JM, Chiang N, Arita M, Serhan CN (2007) Resolvin E1 and protectin D1 activate inflammation-resolution programmes. Nature 447: 869-874. [Crossref]

Correspondence to: Rajiv Saini, Associate Professor, Department of Periodontology and Oral Implantology, Pravara Institute of Medical SciencesLoni, Maharashtra, India;E-mail:drperiodontist@yahoo.co.in, drrajivsaini@gmail.com

Received: February 12, 2016; Accepted: February 22, 2016; Published: February 26,2016 
5. Serhan CN, Chiang N (2004) Novel endogenous small molecules as the checkpoint controllers in inflammation and resolution: Entrée for resoleomics. Rheum Dis Clin North Am 30: 69-95. [Crossref]

6. Van Dyke TE (2008) The Management of Inflammation in Periodontal Disease. JPeriodontol 79: 1601-1608.
7. Hasturk H, Kantarci A, Ohira T, Arita M, Ebrahimi N, et al. (2006) RvE1 protects from local inflammation and osteoclast-mediated bone destruction in periodontitis. FASEB 20: 401-403. [Crossref]

Copyright: (C2016 Saini R. This is an open-access article distributed under the terms of the Creative Commons Attribution License, which permits unrestricted use, distribution, and reproduction in any medium, provided the original author and source are credited. 